\title{
Comparison of Detailed Electron Fluid Model Formulations for Non-equilibrium Plasma Simulations
}

\author{
Jeremiah Boerner* and Iain D. Boyd ${ }^{\dagger}$ \\ Department of Aerospace Engineering, University of Michigan, Ann Arbor, MI, 48109
}

\begin{abstract}
The plasma sheath near a probe surface is a non-neutral, non-equilibrium flow. Two implementations of a non-neutral detailed model electron fluid are compared in this study. Both formulations derive from the electron conservation laws and the electrostatic Poisson equation, but differ in the usage of the Poisson equation. The first implementation (detailed formulation) uses the Poisson equation to compute electron density from the plasma potential. The second implementation (Poisson-consistent formulation) explicitly solves the Poisson equation for potential. Both formulations are incorporated as part of a hybrid fluid Particle In Cell computational code, which is used for simulation of the axisymmetric plasma flow in the sheath near a Faraday probe. The two implementations produce markedly different results for the extent of the sheath. The Poissonconsistent formulation is in good agreement with the collisionless Bohm sheath solution, but the detailed formulation predicts more compact sheaths. The Poisson-consistent formulation is more computationally sensitive: the ion distribution must be primarily at speeds greater than the Bohm velocity to avoid spurious pseudo-presheath structures. The Poisson-consistent formulation also requires nearly $50 \%$ more time than the detailed formulation to complete a simulation. Differences between the Poisson-consistent formulation and the detailed formulation may stem from differing techniques to compute a non-linear term in electron density.
\end{abstract}

\section{Nomenclature}

$\begin{array}{ll}e & =\text { electron charge } \\ \mathbf{E} & =\text { electric field } \\ \mathbf{j} & =\text { electron current density } \\ k_{B} & =\text { Boltzmann constant } \\ L_{D} & =\text { Debye length } \\ M & =\text { Mach number with respect to Bohm velocity } \\ m_{e} & =\text { electron mass } \\ m_{i} & =\text { ion mass } \\ n_{e} & =\text { electron number density } \\ n_{i} & =\text { ion number density } \\ P_{e} & =\text { electron pressure } \\ T_{e} & =\text { electron temperature } \\ T_{i} & =\text { ion temperature } \\ v_{B} & =\text { Bohm velocity } \\ \mathbf{v}_{\mathbf{e}} & =\text { electron velocity } \\ v_{d} & =\text { electron drift velocity } \\ v_{o} & =\text { electron inlet velocity } \\ v d f(v) & =\text { electron velocity distribution function } \\ \mathcal{E}_{0} & =\text { permittivity of free space } \\ \kappa & =\text { thermal conductivity } \\ \sigma & =\text { electrical conductivity } \\ \phi & =\text { local potential } \\ \Psi & =\text { electron potential function }\end{array}$

\footnotetext{
"Graduate Student, Aerospace Engineering (jboerner@umich.edu).

† Professor, Aerospace Engineering (iainboyd@umich.edu).
} 


\section{Introduction}

$\mathrm{E}^{1}$ LECTRIC PROPULSION (EP) systems are favorable for long-duration spacecraft missions due to their high specific impulse. Electrostatic devices, such as ion engines or Hall thrusters, attain much higher values of specific impulse than conventional chemical engines, but at much lower thrust. The efficiency of an EP system can reduce the propellant mass required for a fixed mission profile, or extend the operational lifetime for a fixed propellant mass. The small thrust increments and scalability of EP systems can also be useful for applications that require precision maneuvering.

Ongoing development of Hall thrusters and ion engines includes life tests, performance evaluation, and spacecraft integration. The main failure modes in electrostatic systems stem from ion erosion of key components, such as the discharge chamber walls in Hall thrusters or the ion optics in ion thrusters. Possible impingement of high-energy ions on the spacecraft is an important consideration in the use of EP systems. Experimental measurements of the plasma plume characterize the shape of the plume and its energy spectrum and particle flux, which can be used to predict sputter and deposition rates.

Several diagnostic instruments are commonly used in these experiments, including Faraday probes, Langmuir probes, emissive probes, and retarding potential analyzers. ${ }^{1-4}$ These immersed probes introduce both physical obstructions and electrostatic sheaths, which make it difficult to recover undisturbed plasma properties from the measurements. Experimental tests performed in vacuum chambers must also contend with an excess of background pressure due to pumping limitations. ${ }^{5}$ Collisions with the neutral background gas can lead to an overabundance of low energy charge exchange (CEX) ions which adds further difficulty to interpreting the measurements.

Previous numerical simulation studies suggest that the 1D Bohm sheath solution is reliable over a range of ambient plasma conditions, and that experimental techniques accurately deduce undisturbed plasma properties. ${ }^{6-7}$ These simulations are limited by use of the Boltzmann model for the electron fluid, since typical EP thruster plumes do not satisfy the assumptions that electrons are isothermal, currentless, collisionless, and unmagnetized. Further studies using a detailed model electron fluid indicate that the Bohm sheath solution may over-estimate the size of the sheath, but measurements at the probe surface would still be consistent with the undisturbed plasma properties. ${ }^{8}$

This paper continues the development of the detailed model by describing an alternative approach to solving the system of equations. The original formulation and new Poisson-consistent formulation are described first, with emphasis on the differences in the solution process and the required boundary conditions. The hybrid fluid PIC computational code is then described briefly, along with the numerical parameters of the simulation. Results from both formulations are then compared and discussed.

\section{Electron Fluid Model Formulations}

Two implementations of the detailed model electron fluid are developed here. Both formulations derive from the approach described by Boyd and Yim, ${ }^{9}$ except that plasma neutrality is not assumed. In many experiments a negative bias potential is applied on diagnostic probe surfaces to repel the higher mobility electrons, leading to a non-neutral sheath region near the surface of the probe. The Poisson equation is used to close the equation set by relating the ion and electron densities through the plasma potential.

Both forms of the detailed model begins with the basic electron conservation equations: ${ }^{10}$

Continuity: $\frac{\partial}{\partial t}\left(n_{e}\right)+\nabla \cdot\left(n_{e} \mathbf{v}_{\mathbf{e}}\right)=0$

Momentum: $\frac{\partial}{\partial t}\left(m_{e} n_{e} \mathbf{v}_{\mathbf{e}}\right)+m_{e} n_{e} \mathbf{v}_{\mathbf{e}} \cdot \nabla \mathbf{v}_{\mathbf{e}}=-e n_{e} \mathbf{E}-\nabla P_{e}+\frac{e n_{e} \mathbf{j}}{\sigma}$

Energy: $\quad \frac{\partial}{\partial t}\left(\frac{3}{2} n_{e} k_{B} T_{e}\right)+\frac{3}{2} n_{e} k_{B} \mathbf{v}_{\mathbf{e}} \cdot \nabla T_{e}=\mathbf{j} \cdot \mathbf{E}-P_{e} \nabla \cdot \mathbf{v}_{\mathbf{e}}+\nabla\left(\kappa \nabla T_{e}\right)-3 \frac{m_{e}}{m_{i}} n_{e} v_{e} k_{B}\left(T_{e}-T_{i}\right)$

These are simplified by assuming by assuming a steady state and neglecting inertial terms. The electron pressure $P_{e}$ can be expressed in terms of density and temperature by assuming an ideal gas state relation. The electric field is 
rewritten in terms of the plasma potential as $\mathbf{E}=-\nabla \phi$. The electrical conductivity $\sigma$, thermal conductivity $\kappa$, and collision frequency $v_{e}$ are calculated from basic definitions.

The continuity equation can then be written as a Laplace equation by introducing a potential function $\Psi$ defined as $\nabla \Psi=n_{e} \mathbf{v}_{\mathrm{e}}$ so that continuity reduces to

$$
\nabla^{2} \boldsymbol{\Psi}=0
$$

The energy equation is rearranged into a Poisson equation for the electron temperature. This can be treated as a constant-coefficient equation during computational iteration.

$$
\nabla^{2} T_{e}-\frac{1}{\kappa}\left[\frac{3}{2} n_{e} k_{B} \mathbf{v}_{\mathbf{e}} \cdot \nabla T_{e}+\mathbf{j} \cdot \nabla \phi+n_{e} k_{B} T_{e} \nabla \cdot \mathbf{v}_{\mathbf{e}}-\nabla \kappa \nabla T_{e}+3 \frac{m_{e}}{m_{i}} n_{e} k_{B} v_{e}\left(T_{e}-T_{i}\right)\right]=0
$$

The electron momentum equation can be rearranged to the generalized Ohm's law form of Eq. 4, and the divergence of $\mathbf{j}$ represents conservation of charge at the steady state.

$$
\begin{gathered}
\mathbf{j}=\sigma\left[\frac{1}{e n_{e}} \nabla\left(n_{e} k_{B} T_{e}\right)-\nabla \phi\right] \\
\nabla \cdot \mathbf{j}=0
\end{gathered}
$$

The electrostatic Poisson equation completes the set by relating the ion and electron densities to the potential.

$$
\nabla^{2} \phi=-\frac{e}{\varepsilon_{0}}\left(n_{i}-n_{e}\right)
$$

The two formulations separate at this point, based on how the differential equation resulting from Eq. 5 is solved. The expression can be arranged to solve either for potential or for electron density. The remaining variable is then found through manipulation of the Poisson equation. Although either approach should be valid at a fully selfconsistent steady state, there are computational difficulties in reaching the steady state from some initial state. The procedure and drawbacks of each method are described next.

\section{A. Detailed Formulation}

The original detailed model process solves Eq. 5 for the plasma potential. Combining Eqs. 4-5, expanding, and regrouping the terms by potential gives the form in Eq. 7. This expression can also be treated as a constantcoefficient Poisson equation during iteration.

$$
\nabla^{2} \phi+\frac{\nabla \sigma}{\sigma} \cdot \nabla \phi-\frac{k_{B}}{e}\left\{\nabla^{2} T_{e}+\frac{\nabla \sigma}{\sigma} \cdot \nabla T_{e}+\left(T_{e} \frac{\nabla \sigma}{\sigma}+\nabla T_{e}+T_{e} n_{e} \nabla \frac{1}{n_{e}}\right) \cdot \frac{\nabla n_{e}}{n_{e}}+T_{e} \frac{\nabla^{2} n_{e}}{n_{e}}\right\}=0
$$

The electrostatic Poisson equation is then manipulated to give the electron density in terms of the ion density and the newly calculated potential field $\phi$ according to

$$
n_{e}=n_{i}+\frac{\varepsilon_{0}}{e} \nabla^{2} \phi
$$

The electron density, electron temperature, and plasma potential are all coupled, so Eqs. 3, 7, and 8 must be solved iteratively.

The difficulty in this process lies in accurately determining the electron density. Firstly, there is a resolution difficulty due to the vast disparity in scales between the Laplacian of potential and differences in number density. The electron density changes several orders of magnitude over a corresponding potential change of just a few volts. Secondly, the ion density derives from simulated particles and is subject to significant statistical scatter in the PIC 
simulation. As a consequence, the instantaneous values of ion density are not suitable for use in calculating the electron density. A running average of ion density is required to perform this calculation.

Boundary conditions for this approach are straightforward. The potential (or its gradients) need to be specified at the probe and along free surfaces. Likewise, properties for the PIC ions need to be assigned at the inlets. The electron density requires no constraints since it is not solved from a differential equation.

\section{B. Poisson-consistent Formulation}

This alternative technique solves Eq. 5 for electron density. The form in Eq. 9 is obtained by combining and rearranging Eqs. 4-5. This form is slightly more difficult to solve, due primarily to the non-linear term that appears in the coefficient of $\nabla n_{e}$.

$$
\nabla^{2} n_{e}+\left(\frac{\nabla \sigma}{\sigma}+\frac{\nabla T_{e}}{T_{e}}+n_{e} \nabla \frac{1}{n_{e}}\right) \cdot \nabla n_{e}+\left[\frac{\nabla^{2} T_{e}}{T_{e}}+\frac{\nabla \sigma}{\sigma} \cdot \frac{\nabla T_{e}}{T_{e}}-\frac{e}{k_{B} T_{e}}\left(\frac{\nabla \sigma}{\sigma} \cdot \nabla \phi+\nabla^{2} \phi\right)\right] n_{e}=0
$$

Once the electron density is found, the electrostatic Poisson equation is then solved for the potential using the original form in Eq. 6. The electron density, electron temperature, and plasma potential remain coupled, so Eqs. 3, 6, and 9 require an iterative solution.

This process mostly avoids the difficulties of the original detailed model technique: the ion and electron densities are of comparable magnitudes, and solving the Poisson equation as a differential equation can accommodate statistical scatter in the ion density. Equation 9 requires significantly more iterations to converge however, due to the non-linear term.

The boundary conditions ultimately prove to be the greatest difficulty in applying this approach. Appropriate boundary conditions for the electron density must be identified and enforced. Furthermore, these have to be consistent with the boundary conditions on the potential. The best results are obtained by using the Robin or thirdkind boundary conditions for both the potential and electron density at the inlet. At the probe, the potential is simply the bias voltage. The electron density at the probe is calculated via integration of the potential-shifted velocity distribution, as follows.

Electrons at the inlet are assumed to follow a Maxwellian velocity distribution function $v d f\left(v_{e}\right)$. As an electron fluid element moves into the domain, it is assumed to interact only with the electric potential, so that the total energy remains constant and the local velocity $v(\phi)$ of the fluid element depends only on its initial velocity $v_{o}$ and the local potential $\phi$ as

$$
v(\phi)=\sqrt{v_{o}^{2}-\frac{2 e}{m_{e}}|\phi|}
$$

Large values of $v_{o}$ are not affected much, while small values of $v_{o}$ may change significantly. For example, Eq. 10 results in an imaginary velocity if the local potential is sufficiently large (i.e., sufficiently negative) compared to $v_{o}$. Such a result indicates that the electron fluid element with that initial velocity has been completely repelled and does not reach that local potential.

Since a fluid element interacts only with the potential, the phase space density is constant along a trajectory. That is, the inlet value of $v d f\left(v_{o}\right)$ is carried along with the fluid element as the position and velocity change: $v d f(v(\phi))=$ $v d f\left(v_{o}\right)$. The potential-shifted velocity distribution $v d f(v(\phi))$ can then be integrated numerically to obtain the local electron number density. This is performed separately from the rest of the Poisson-consistent formulation to provide boundary values of electron density at the probe surfaces.

The Poisson-consistent formulation is also sensitive to the inlet velocity distribution of the ions. If the ion velocity distribution function has a significant fraction at speeds less than the Bohm velocity, the simulation will develop a pre-sheath-like structure that effectively overrides the potential and electron density boundary conditions applied at the inlet. This is perhaps not entirely surprising, since the Bohm velocity is the minimum ion velocity required to obtain purely real (non-oscillating) solutions of the Bohm sheath. In the context of these simulations however, the apparent pre-sheath structure reflects the mathematical incompatibility of the ion velocity, rather than physically realistic behavior. 


\section{Computational Model Description}

The computational model simulates 2D axisymmetric flow using a hybrid fluid Particle In Cell (PIC) method. Ions and neutrals are treated with a PIC model, ${ }^{11}$ while electrons and fields are simulated with one of the fluid model formulations described previously. An alternating direction implicit (ADI) solver is used to solve the differential equations as necessary. A direct simulation Monte Carlo (DSMC) routine ${ }^{12}$ is in place to handle collisions, although the plasma conditions in this study are very nearly collisionless. Simulated particle weight is increased in steps from the centerline to the outer edge of the domain in order to reduce the number of simulated particles and reduce the total computation time. Macroscopic properties are determined from weighted averages of the particles within a cell.

At the upstream and outer radial edges, inlet boundary cells generate the ion and neutral particles that enter the domain during each time step. The velocity of each particle is selected using an acceptance/rejection method from a Maxwellian distribution, with drift velocity and thermal temperature as inputs. Properties are sampled from separate distributions for each component species in simulations with more than one inflowing species. When particles collide with probe surfaces, they undergo diffuse reflection and are converted to neutral particles. Charged particle collisions with the "collecting surface" are recorded and weighted by the area of the impacted cell edge. Summing these area-weighted collisions over the collecting surface gives the simulated collected current. The collected ion and electron currents are averaged during the sampling process along with other macroscopic properties.

Robin or third-kind boundary conditions are applied on potential and electron density along the upstream edge. At the outer radial edge, the gradient of electron density is held constant, and the gradient of potential is set to zero. The potential is set to a single bias voltage over all the probe faces. For the Poisson-consistent formulation, the electron density is assigned different values on the probe ram face and side face as calculated from integration of the potential-shifted velocity distribution.

\section{Inflow Conditions and Computational Grid}

A typical EP plume observed in an experimental vacuum chamber has several intermixed particle populations. The core of the plume consists of a high-velocity beam population formed and accelerated in the thruster. A charge exchange (CEX) population is likely to be present as well, formed by collisions downstream of the thruster's main acceleration region. Multiple charge ions may also occur as part of the beam and CEX components. Neutral particles can be introduced as propellant escapes the thruster without being ionized, or from the rest of the chamber if the exhaust is not fully evacuated by the pumps or condensed on the walls.

In this study we first consider two test cases that consist of a single population of ions that differ only in temperature: a "cold CEX" case with $T_{i}=300 \mathrm{~K}$ and a "hot CEX" case with $T_{i}=11,600 \mathrm{~K}(1 \mathrm{eV})$. The test cases are a baseline for comparison with the Bohm sheath solution, and for demonstrating the effect of ion temperature on the simulation. We then consider a "composite" population that is more representative of an EP plume, with CEX, beam, and double charge ion populations. The CEX population in the composite simulation is the same as the hot CEX test case population.

Freestream plasma properties are representative of the flow downstream of a low power Hall thruster. In particular, we choose properties corresponding to the ion current measured $50 \mathrm{~cm}$ downstream and $75^{\circ}$ off-axis in the exhaust plume of a Busek Co. "BHT-200" 200 W xenon Hall thruster. This is a region of interest where CEX ions represent the majority of the ion flux. In the composite simulation, CEX ions are assumed to account for $75 \%$ of the current, and beam ions the

Table 1. Distribution properties and characteristic dimensions for single beam and composite cases.

\begin{tabular}{lccrcc}
\hline \hline Test cases & $\begin{array}{c}n_{i} \\
10^{14} \mathrm{~m}^{-3}\end{array}$ & $\begin{array}{c}v_{i} \\
\mathrm{~m} / \mathrm{s}\end{array}$ & \multicolumn{1}{c}{$\begin{array}{c}T_{i} \\
\mathrm{~K}\end{array}$} & $\begin{array}{c}L_{D} \\
\mathrm{~mm}\end{array}$ & $M$ \\
\hline Cold CEX & 1.2951 & 1,026 & 300 & 0.653 & 1.20 \\
Hot CEX & 1.2951 & 1,026 & 11,600 & 0.653 & 1.20 \\
Electrons & 1.2951 & 1,026 & 11,600 & 0.653 & 1.20 \\
\hline
\end{tabular}

\begin{tabular}{lcrrrr} 
Composite & $\begin{array}{c}n_{i} \\
10^{14} \mathrm{~m}^{-3}\end{array}$ & $\begin{array}{c}v_{i} \\
\mathrm{~m} / \mathrm{s}\end{array}$ & $\begin{array}{c}T_{i} \\
\mathrm{~K}\end{array}$ & $\begin{array}{c}L_{D} \\
\mathrm{~mm}\end{array}$ & $M$ \\
\hline Beam & 0.4316 & 2,381 & 11,600 & 1.132 & 2.78 \\
CEX & 1.2951 & 1,026 & 11,600 & 0.653 & 1.20 \\
Double & 0.0678 & 1,930 & - & 2.018 & 2.26 \\
Electron fluid & 1.8623 & 1,406 & 11,600 & 0.551 & 1.64 \\
\hline \hline
\end{tabular}
remaining $25 \%$.

Double charge ions are selected at the same ratio from the CEX and beam populations to account for $10 \%$ of the total current flux. The double charge ions have a drift velocity that is approximately a factor of $\sqrt{2}$ larger than the single charge ion drift velocities, so the double charge ion density is proportionally lower to maintain the same total current flux. There is not a well-defined temperature, since the double charge ions are drawn from both the beam and CEX distributions. Properties of the ion components are reported in Table 1. 
The inflowing plasma is neutral and currentless, so the electron fluid at the inlet has the same charge density and mean velocity as the ion components. For the composite case the electron fluid properties are equal to the bulk properties of all three ion components combined. The electron temperature is set at $1 \mathrm{eV}(11,600 \mathrm{~K})$ at the inlet and at the probe surfaces.

Plasma potential in the freestream is set at $0 \mathrm{~V}$. In keeping with standard experimental practice, the probe is set to a uniform potential, $-5 \mathrm{~V}$ for the cases described here.

The cylindrical Faraday probe geometry is well suited to a computational grid consisting of equally spaced rectangular cells. The computational domain is shown schematically in Fig. 1, with the probe front surface divided into collecting surface and guard ring.

A Bohm sheath solution for the beam ions provides an estimate of the required domain size, suggesting an upstream length of $1.1 \mathrm{~cm}\left(10 L_{D}\right)$ for these conditions. The appropriate radial extension for the probe geometry is not readily apparent, and is set to one quarter-radius beyond the probe edge. Previous experience with the computational code suggests that the maximum cell spacing should be at least a factor of 12 smaller than the Debye length based on the total charge density. That Debye length is $0.551 \mathrm{~mm}$ for the composite case, and the cells are dimensioned conservatively at $0.04 \mathrm{~mm}$ on a side. The final geometry extends 390 cells $(1.560 \mathrm{~cm})$ along the probe axis and 390 cells $(1.560 \mathrm{~cm})$ radially, with 238 elements $(0.952 \mathrm{~cm})$ along the collecting surface and 80 elements $(0.320 \mathrm{~cm})$ along the guard ring. Altogether there are 112,350 cells outside of the probe body. Dimensions of the probe front face are selected to match the experimental instrument employed in Ref. 13.

The simulation time step is selected so that the fastest ions travel less than one cell length per iteration. For the beam population, ions that enter at twice the thermal speed beyond the drift velocity arrive at the probe with a velocity of $6,220 \mathrm{~m} / \mathrm{s}$. Dividing the cell length by this speed and rounding down sets the time step at $5 \times 10^{-9} \mathrm{~s}$.

Each probe simulation is run for 10,000 iterations to reach a steady state, followed by 20,000 sampled iterations. At steady state the particle counts are approximately 1.6 million particles for the single beam case and 2.6 million particles for the composite case. The particle count does not vary significantly between the detailed formulation and the Poisson-consistent formulation. The detailed formulation requires slightly more than 26 hours to run the composite case on a $3.8 \mathrm{GHz}$ Pentium 4 desktop system. The Poisson-consistent formulation is significantly slower, requiring almost 38 hours on the same computer.

\section{Results}

The initial two test cases are useful for investigating the impact of ion temperature on the simulation and comparing the Poisson-consistent formulation results with the Bohm sheath solution. For both the cold CEX and hot CEX cases the drift velocity is $1026 \mathrm{~m} / \mathrm{s}$ and the Bohm velocity is $855 \mathrm{~m} / \mathrm{s}$, yielding a Bohm velocity Mach number of 1.20. Figure 2 shows the velocity distribution functions for the ion populations in both cases. The thermal variation in particle speeds is such that about $11 \%$ of the cold CEX and $42 \%$ of the hot CEX ions are at speeds less than the Bohm velocity.

As mentioned previously, the Poisson-consistent formulation is sensitive to ions that do not meet the Bohm criterion. Large oscillations in the potential and density fields are noted during early iterations in both test cases. The oscillations decay rapidly within the first two thousand iterations, and further iterations appear to proceed monotonically toward a steady state. The initial overshoot in potential is sufficiently large that the electric field expels many of the ion particles in the first few cells back out the inlet. When the potential then swings below the boundary value, the electric field accelerates the remaining ion particles in those cells toward the probe. This combination creates a potential drop of $\sim 0.15 \mathrm{~V}$ and a region of ion density that is $8-10 \%$ lower than the inlet density.

In the cold CEX case, most ion particles enter with a relatively large velocity and the potential drop causes only a small increase in velocity. The ion density recovers as new ion particles enter the domain, and the potential drop diffuses. In the hot CEX case however, many ion particles enter at a low velocity and experience a large increase in velocity. The slower particles are constantly accelerated into the domain, and the low ion density region and potential drop persist. Although the final state resembles a presheath, the structure is in fact a residual of the initial computational oscillation. The term "pseudo-presheath" is used to describe this feature.

Profiles of the plasma properties in the sheath are generated from the simulation data by averaging over the first 100 cells $(4 \mathrm{~mm})$ from the centerline at each axial position upstream of the front probe face. Radial average profiles of potential, electron density and ion density are shown for the test cases in Figs. 3-5. For the cold CEX case, the Poisson-consistent formulation produces results that are in excellent agreement with the Bohm sheath solution. The hot CEX case shows a distinct pseudo-presheath structure, but still follows the trends of the Bohm sheath. The agreement improves near the probe surface as the potential and electron density approach fixed boundary values. 
The composite case has three component populations, and as seen in Fig. 6, each population has a fraction of particles at speeds less than the Bohm velocity. By component, $42 \%$ of the hot CEX particles, $11 \%$ of the double charge particles, and $3.7 \%$ of the beam particles are slower than the Bohm velocity. The additional beam and double charge ions help stabilize the iteration, since the majority of those particles are not significantly affected by the initial potential oscillation. In this case though, the hot CEX makes up too much of the total population and a pseudo-presheath occurs. The pseudo-presheath is smaller in this case, with the potential drop decreased to $\sim 0.05 \mathrm{~V}$, and the total density about 5-7\% lower than the inlet density.

The potential is in passable agreement with the Bohm sheath profile over much of the domain in Fig. 7. The electron density in Fig. 8 shows a larger discrepancy. This can be explained in part by noting that the plasma remains neutral for several millimeters after it enters the domain. Since the total ion density is decreased near the inlet as shown in Fig. 9, the electron density is likewise decreased. Another interesting feature is that the ion components are essentially independent. The beam and double charge components form sheaths that match the corresponding Bohm sheath solutions quite well. Although the hot CEX component forms a pseudo-presheath, it has little impact on the behavior of the faster ion components.

Results obtained by using the detailed formulation on the composite case are also shown in these figures. Previous simulations using this formulation demonstrated the same departures from the Bohm sheath behavior that are observed here. ${ }^{8}$ Mainly, the detailed formulation produces sheath profiles that do not extend as far from the probe as the Bohm sheath solution suggests. A contour plot of electron density for the cold CEX case using the Poisson-consistent formulation is shown in Fig. 10. The results obtained from the detailed formulation on the same conditions are shown in Fig. 11 for comparison. The Poisson-consistent model shows a thick sheath extending 5-6 $\mathrm{mm}$ from the front probe surface, and a wake-like structure along the probe body. The detailed model results show a thinner sheath that extends no more than $3 \mathrm{~mm}$ from the probe face, and is nearly uniform along the front and side of the probe. This behavior is evident in the potential, electron density, and ion density in all of the cases investigated.

In the previous work this was attributed to variation in the electrical conductivity, which the collisionless Bohm sheath does not take into account. Since both the detailed and Poisson-consistent formulations derive from the same set of governing equations, we could reasonably expect to recover the detailed model results, including the variations in conductivity, with the Poisson-consistent formulation. But instead, the Poisson-consistent formulation appears to be in good agreement with the Bohm sheath.

Radial average profiles of the electrical conductivity are considered next, in Fig. 12. The detailed formulation and Poisson-consistent formulation show a similar range of conductivity from the probe surface to the edge of the sheath. Conductivity is primarily a function of electron density in these conditions, and the variation corresponds well with the electron density for both formulations. Both formulations show similar variations in conductivity, but different overall results, indicating that there must be another difference between the two formulations.

The formulations differ primarily in the solution of Eq. 5, aside from a few minor differences elsewhere. The form of the non-linear electron density term is different between the two formulations, and this is conjectured to be the source of the discrepancies. For the detailed formulation, the non-linear term can be computed exactly as it appears on the left-hand side of Eq. 11, using the available values of electron density. In the Poisson-consistent formulation however, the term must be rearranged to the form on the right-hand side in order to be compatible with the iteration procedure of the ADI solver.

$$
\nabla \frac{1}{n_{e}} \cdot \nabla n_{e}=-\frac{1}{n_{e}^{2}} \nabla n_{e} \cdot \nabla n_{e}
$$

Both of these forms of the non-linear term are calculated using the simulated electron density from the cold CEX case results, for both the detailed and Poisson-consistent formulations. The detailed formulation shows two profiles in Fig. 13 for the two forms, indicating that the simulated electron density does not satisfy the equality in Eq. 11. In Fig. 14, the two forms collapse to a single profile for the Poisson-consistent formulation. Taking these results together could imply that the iteration process in the detailed formulation may need to use an alternate form of the

non-linear term. At this point the detailed formulation has not been modified to verify that such a change would cause the results to satisfy Eq. 11, or to assess agreement with the Poisson-consistent formulation results.

\section{Conclusion}

Two formulations of the detailed model were used in hybrid fluid PIC simulations of the plasma flow near a constant potential surface representing a Faraday probe. The Poisson-consistent formulation is found to be sensitive to ion distributions with a significant fraction of particles at speeds less than the Bohm velocity. When such a 
distribution is simulated, a pseudo-presheath structure may form when the ion particles interact with initial oscillations in the solution. Multiple populations do not interact strongly though, so some components may develop normally while another component develops a pseudo-presheath. Results from the Poisson-consistent formulation are generally in good agreement with the Bohm sheath solution.

The detailed formulation derives from the same governing equations as the Poisson-consistent formulation, but produces substantially different results for the same simulation conditions. The sheath thickness is much smaller in the detailed formulation. Each of the ion components behaves normally throughout the domain, regardless of whether the ion particles enter at speeds greater than the Bohm velocity. The conductivity is comparable to the Poisson-consistent formulation results, and is not believed to be the source of discrepancies between the two formulations.

It is conjectured that the differences between the Poisson-consistent formulation and the detailed formulation stem from the treatment of a non-linear term in electron density. Results from the detailed formulation do not satisfy a mathematical identity that appears in the non-linear term. Appropriate revisions to the detailed formulation to test this conjecture have not been performed to date.

\section{References}

1. Fife, J. M., Hargus, W. A., et al., "Spacecraft Interaction Test Results of the High Performance Hall System SPT-140," AIAA-2000-3521, 36th AIAA/ASME/SAE/ASEE Joint Propulsion Conference, July 2000.

2. Hofer, R. R., Hass, J. M., Gallimore, A. D., "Ion Voltage Diagnostics in the Far-field Plume of a High Specific Impulse Hall Thruster," AIAA-2003-4556, 39th AIAA/ASME/SAE/ASEE Joint Propulsion Conference, July 2003.

3. Linnell, J.A., and Gallimore, A.D., "Internal plasma potential measurements of a Hall thruster using xenon and krypton propellant", Physics of Plasmas, No. 103504, Vol. 13, Issue 10, October 2006.

4. King, L. B., Gallimore, A. D., Maresse, C. M., "Transport-Property Measurements in the Plume of an SPT-100 Hall Thruster," Journal of Propulsion and Power, Vol. 14, No. 3, May-June 1998, pp. 327-335.

5. Walker, M. L. R., Gallimore, A. D., "Hot Flow Pressure Map of a Vacuum Facility as a Function of Flow Rate to Study Facility Effects," IEPC-2003-077, 28th International Electric Propulsion Conference, March 2003.

6. Boerner, J., Boyd, I. D., "Numerical Simulation of Probe Measurements in Non-equilibrium Plasma," AIAA2005-4790, 36th AIAA Plasmadynamics and Lasers Conference, June 2005.

7. Boerner, J., Boyd, I. D., "Numerical Simulation of Faraday Probe Measurements in a Multi-Component Nonequilibrium Plasma," IEPC-2005-085, 29th International Electric Propulsion Conference, November 2005.

8. Boerner, J., Boyd, I. D., "Numerical Simulation of Probe Measurements in a Non-equilibrium Plasma, Using a Detailed Model Electron Fluid," AIAA-2007-995, 45th AIAA Aerospace Sciences Meeting, January 2007.

9. Boyd, I. D. and Yim, J. T., "Modeling of the Near Field Plume of a Hall Thruster," Journal of Applied Physics, Vol. 95, 2004, pp. 4575-4584.

10. Mitchner, M. and Kruger, C. H., Partially Ionized Gases, Wiley, New York, 1973.

11. Birdsall, C. K. and Langdon, A. B., Plasma Physics Via Computer Simulation, Adam Hilger Press, 1991.

12. Bird, G. A., Molecular Gas Dynamics and the Direct Simulation of Gas Flows, No. 42 in Oxford Engineering Science Series, Oxford University Press, 1994.

13. Ma, Tammy, "Quantification with Two-Dimensional Spatial Resolution of the Ion Flux Emitted from a $200 \mathrm{~W}$ Hall Effect Thruster," California Institute of Technology, Pasadena, California, 2004, (unpublished). 


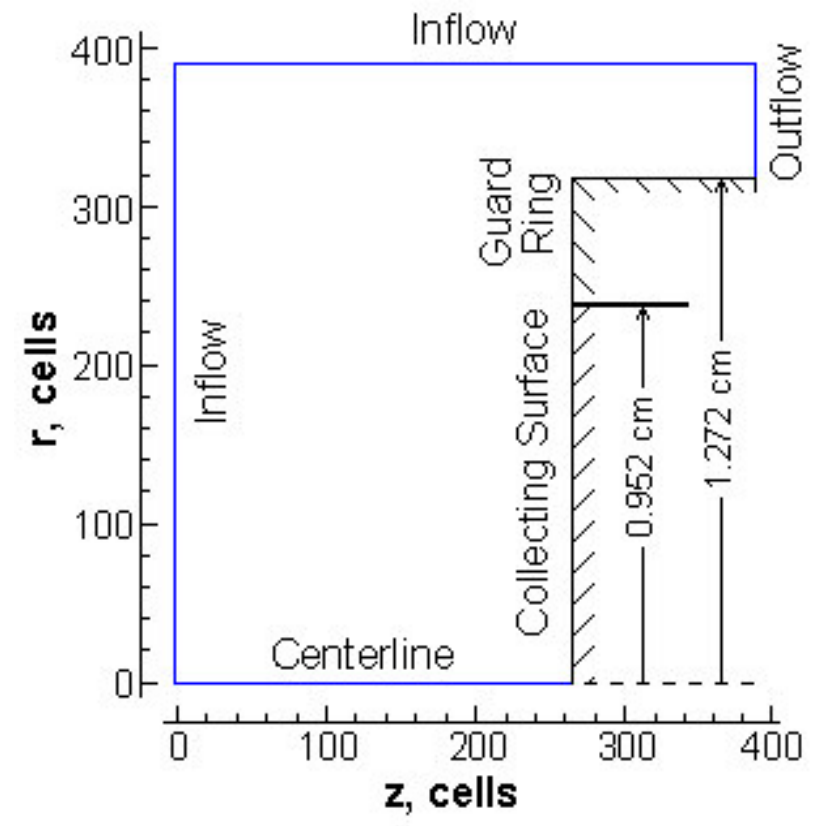

Figure 1. Computational domain, with probe surfaces at the right lower corner. Flow is from left to right.

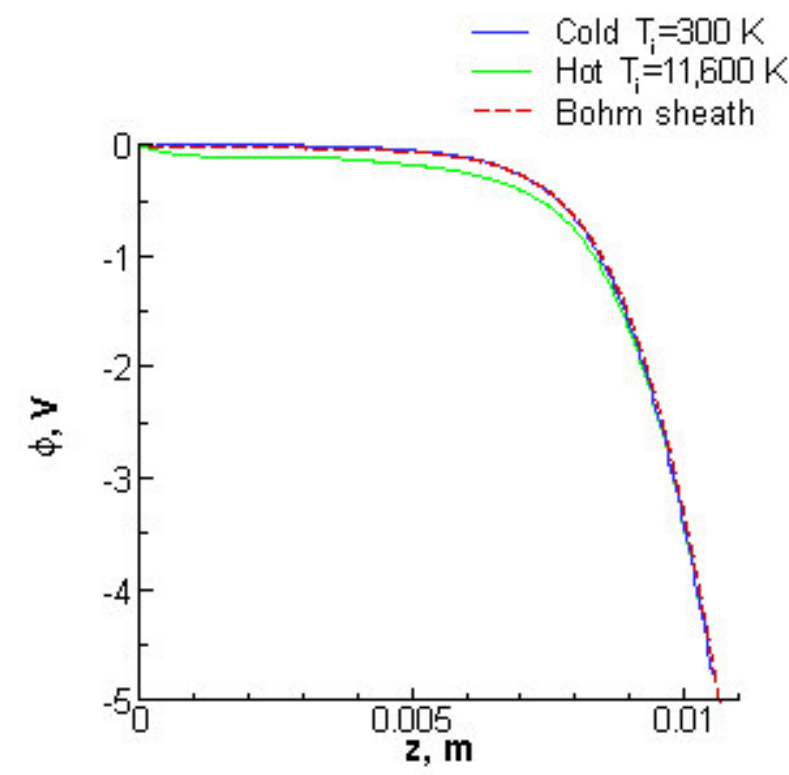

Figure 3. The radial average profile of potential for the hot CEX case demonstrates a pseudo-presheath structure near the inlet. The cold CEX case produces a profile that is in good agreement with the Bohm sheath.

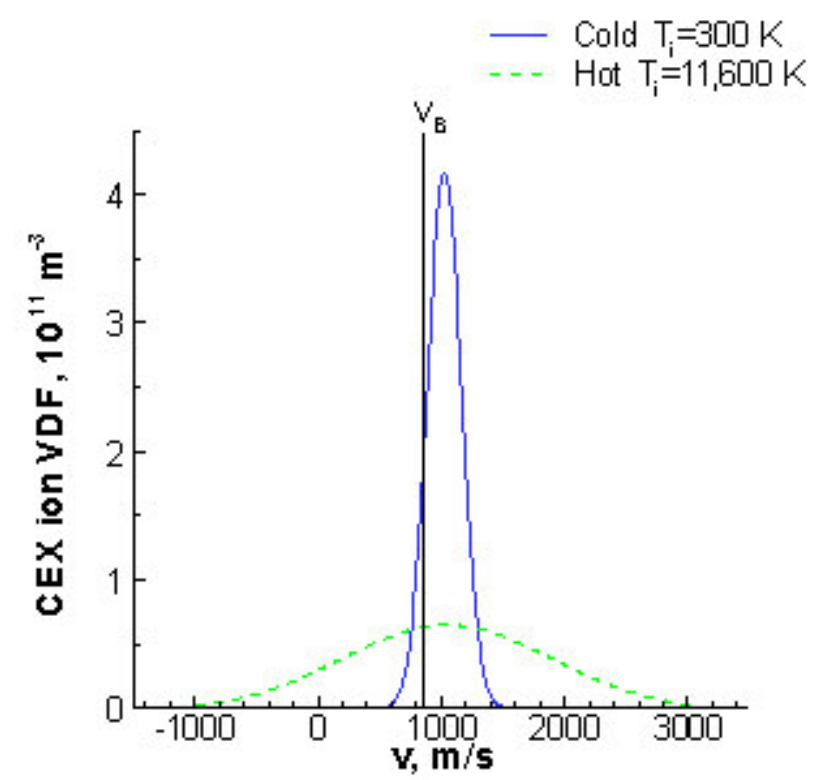

Figure 2. Velocity distribution functions for the cold CEX and hot CEX cases. Nearly half of the hot CEX ions are slower than the Bohm velocity (here $\mathrm{v}_{\mathrm{B}}=855 \mathrm{~m} / \mathrm{s}$.)

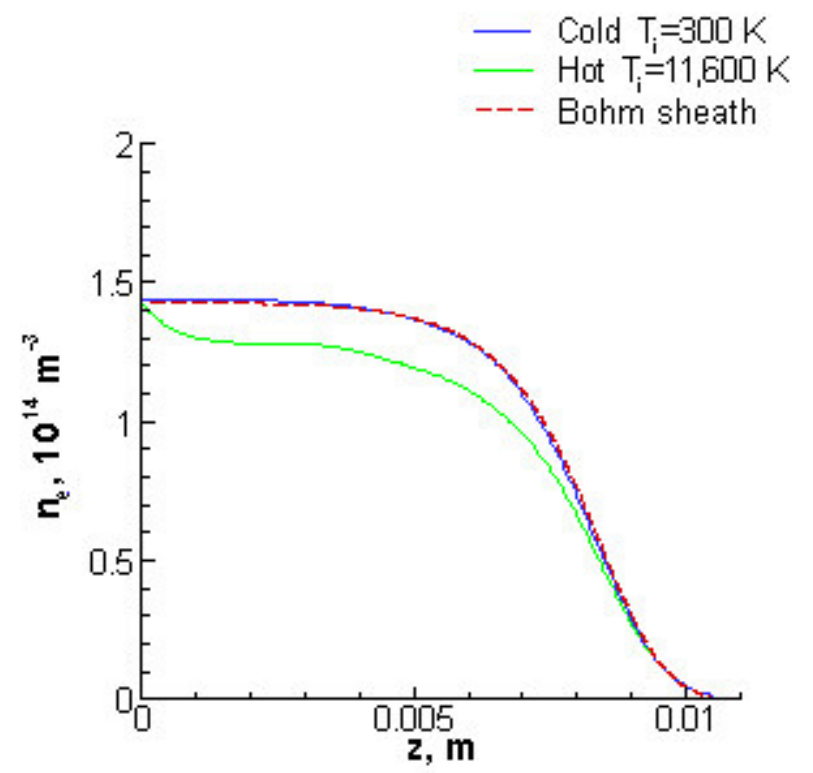

Figure 4. The radial average profile of electron density for the hot CEX case shows a distinct pseudo-presheath structure. 


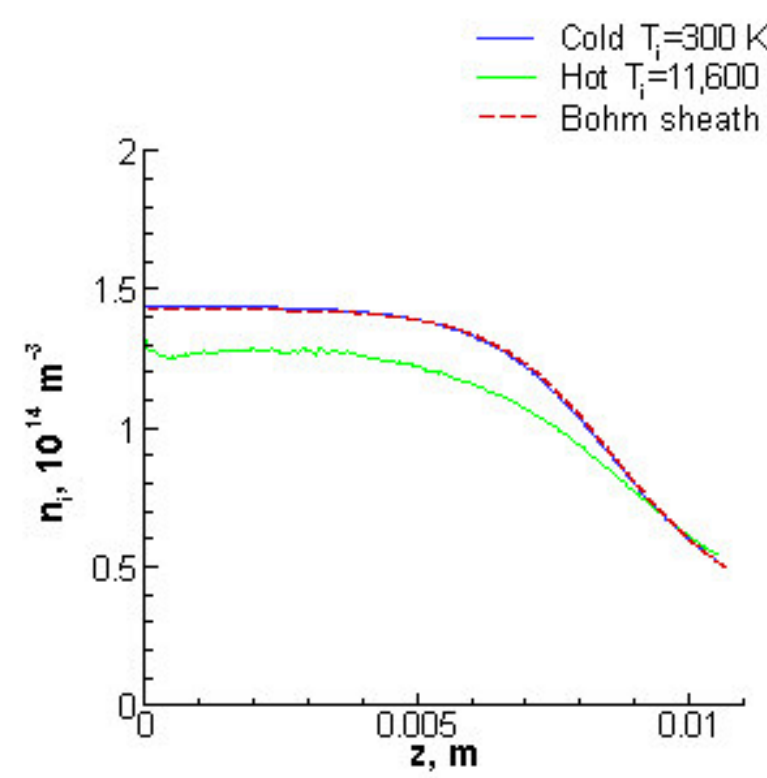

Figure 5. The radial average profile of ion density also shows an effect of the pseudo-presheath. Ions entering the domain in the hot CEX case are immediately accelerated, leading to a lower density since the inflowing flux is conserved.

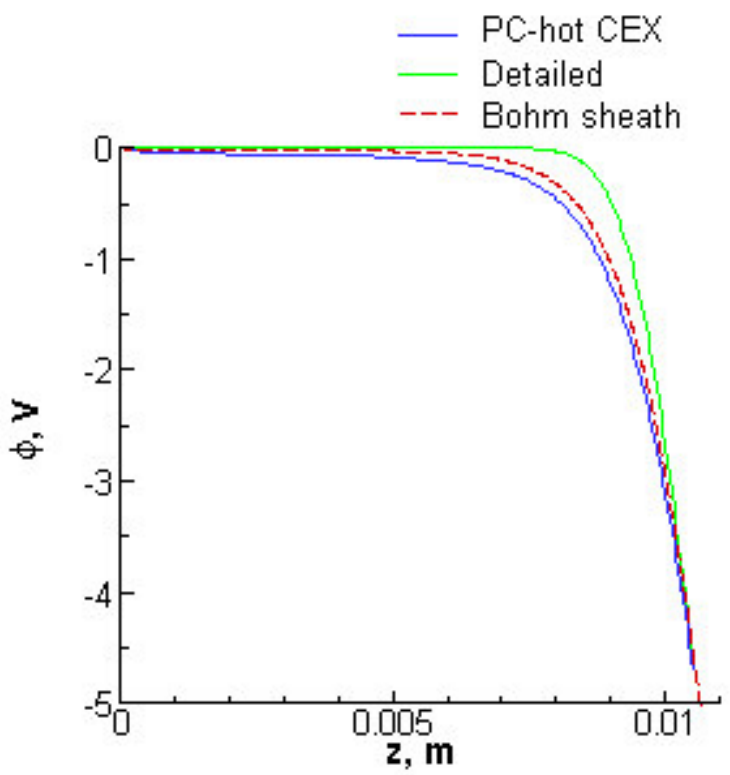

Figure 7. The radial average profiles of simulated potential for the composite case show a disparity between the Poisson-consistent results and the detailed model results.

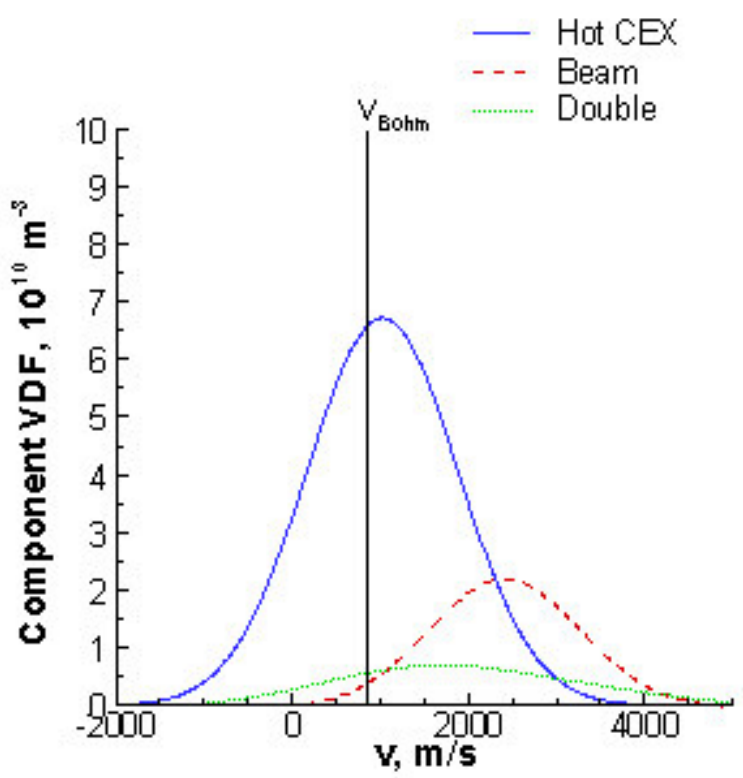

Figure 6. Velocity distribution functions for the ion populations in the composite case. Each population has some fraction at speeds less than the Bohm velocity.

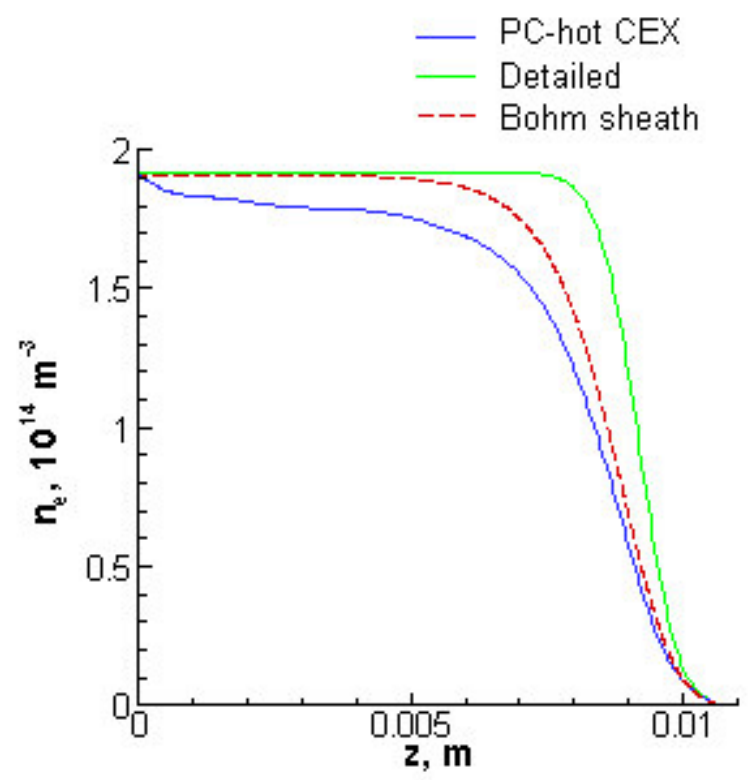

Figure 8. The hot CEX composite case still shows a pseudo-presheath in the radial average profiles of electron density. 


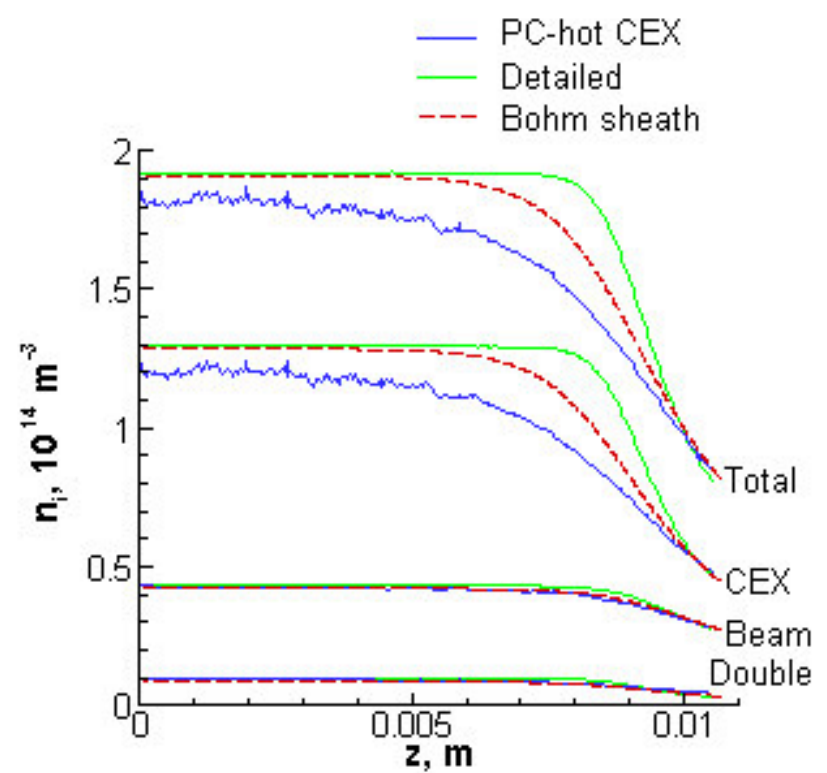

Figure 9. Radial average profiles of simulated ion density for the composite case. Note that the components are independent in both models, and that the pseudo-presheath structure is only evident in the CEX component.

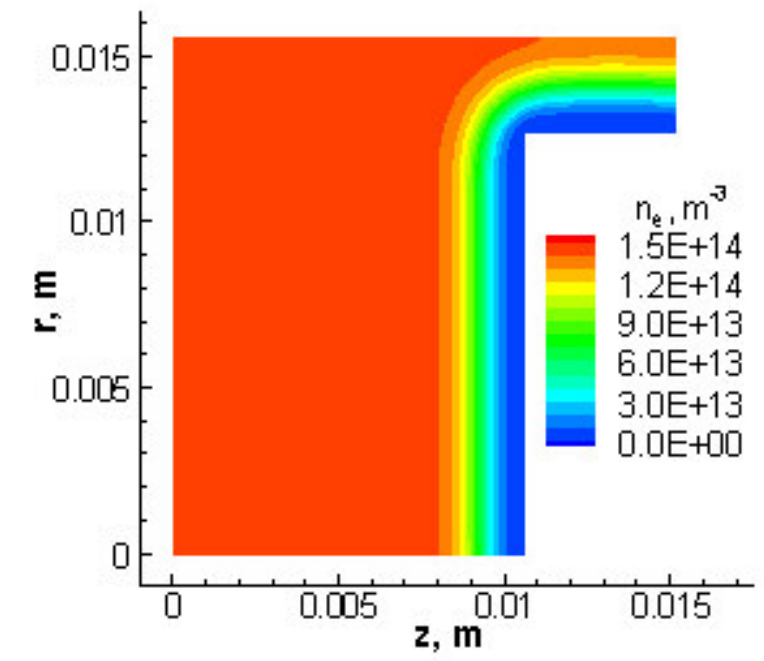

Figure 11. Contours of electron density for the cold CEX case using the detailed model. The sheath is uniform along the front and side of the probe. This sheath is also significantly more compact than the sheath in the Poisson consistent model.

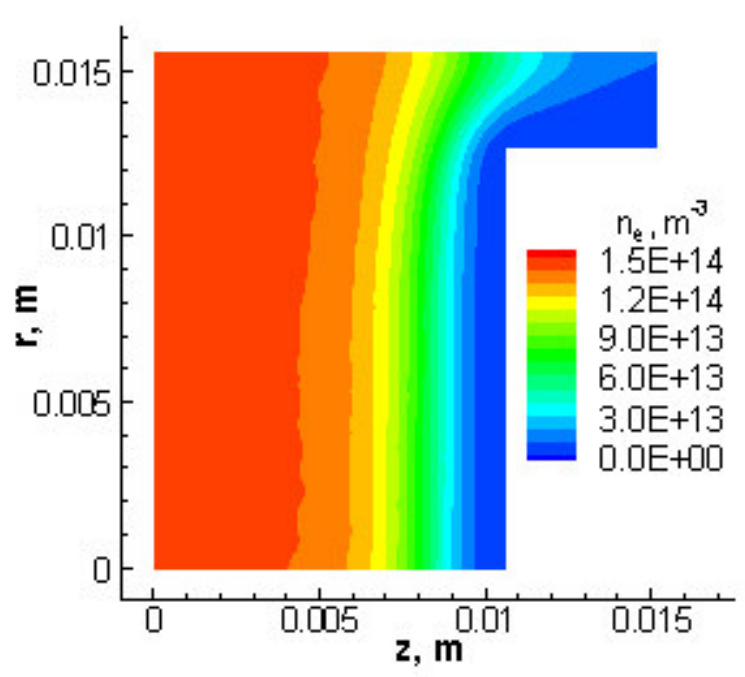

Figure 10. Contours of electron density for the cold CEX case using the Poisson-consistent model. The sheath is uniform over the front of the probe, but shows a wake-like region of low density along the side of the probe.
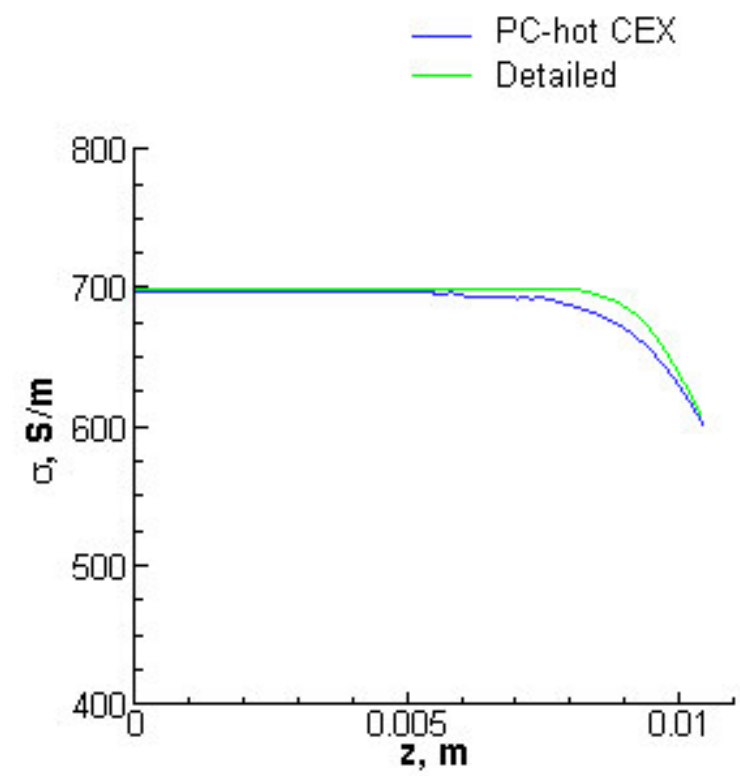

Figure 12. The detailed model and Poisson consistent model produce similar radial average profiles of electrical conductivity. 


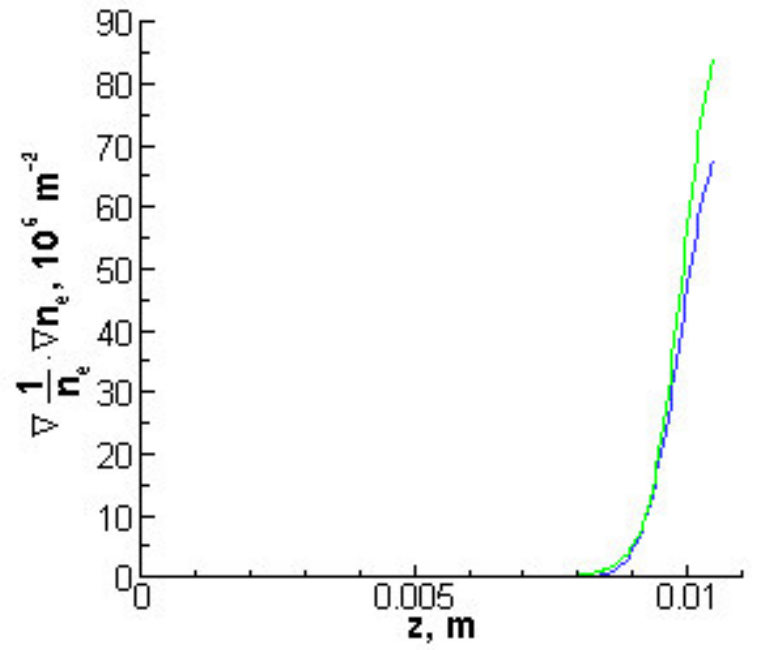

Figure 13. The two forms of the non-linear term presented in Eq. 11 yield different profiles for the detailed formulation results.

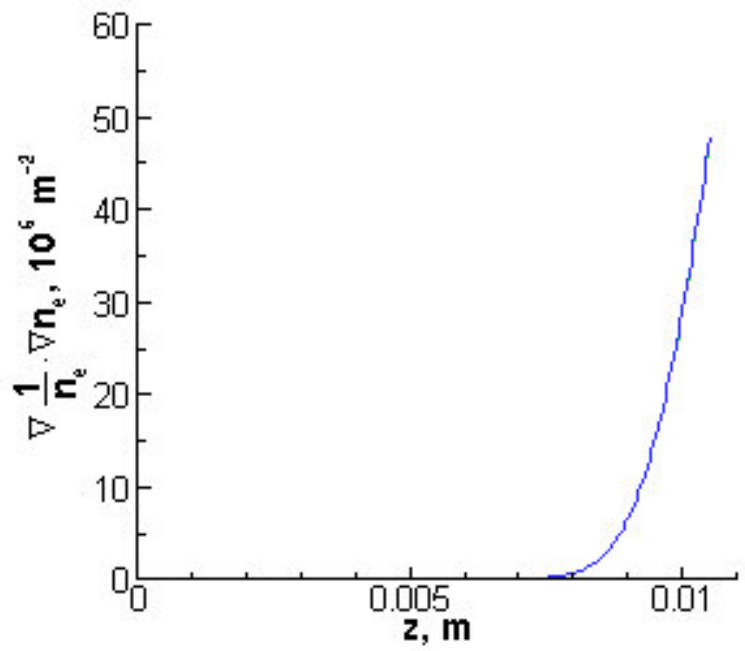

Figure 14. Results from the Poisson-consistent formulation give identical values for both forms of the non-linear term. 